\title{
Le Doyen de Killerine ou l'Art de parler. Discours rapporté et rhétorique chez Prévost
}

\section{Lise Charles}

\section{(2) OpenEdition \\ 1 Journals}

Electronic version

URL: http://journals.openedition.org/rhetorique/809

DOI: $10.4000 /$ rhetorique.809

ISSN: 2270-6909

\section{Publisher}

UGA Éditions/Université Grenoble Alpes

\section{Printed version}

ISBN: 978-2-37747-066-2

\section{Electronic reference}

Lise Charles, "Le Doyen de Killerine ou l'Art de parler. Discours rapporté et rhétorique chez Prévost », Exercices de rhétorique [Online], 12 | 2019, Online since 19 January 2019, connection on 12 September 2020. URL : http://journals.openedition.org/rhetorique/809 ; DOI : https://doi.org/10.4000/rhetorique. 809

This text was automatically generated on 12 September 2020 .

\section{(c) (i) (9)}

Les contenus de la revue Exercices de rhétorique sont mis à disposition selon les termes de la Licence Creative Commons Attribution - Pas d'Utilisation Commerciale - Partage dans les Mêmes Conditions 4.0 International. 


\title{
Le Doyen de Killerine ou l'Art de parler. Discours rapporté et rhétorique chez Prévost
}

\author{
Lise Charles
}

1 Le Doyen de Killerine, publié de 1735 à 1740, marque l'allégeance de l'abbé Prévost aux jésuites, qui accusent alors les romans de constituer un «catéchisme des mauvaises mœurs ${ }^{1}$ ", et qui viennent de s'en prendre plus particulièrement à Cleveland, que Prévost publie par morceaux depuis $1731^{2}$. L'ouvrage se présente, dès son sous-titre, comme une " Histoire morale [...] ornée de tout ce qui peut rendre une lecture utile et agréable ». Une "histoire morale ", en effet. Dans ce "roman jésuite ${ }^{3}$ " écrit à la première personne, le doyen raconte ses efforts constants pour veiller à l'éducation des enfants que son père a eus d'une seconde union, Georges, Patrice et Rose, et leur inspirer une conduite vertueuse.

2 Parmi les ornements susceptibles de rendre une lecture « utile et agréable » figurent sans doute les discours prononcés par les personnages, censés apporter de la variété au récit par les changements de voix et de points de vue qu'ils impliquent. En vérité, les différents acteurs du Doyen de Killerine parlent souvent, longuement, et c'est surtout la longueur des discours rapportés qui fait celle de ce vaste roman en six parties. Beaucoup de ces discours sont des morceaux d'éloquence, structurés et qualifiés comme tels, si bien que le roman ne s'apparente pas seulement à un manuel de casuistique (Jean Sgard le rapproche du Dictionnaire des cas de conscience de Pontas, avec ses exemples numérotés ${ }^{4}$ ), mais à un long manuel de rhétorique contenant une collection d'exemples ${ }^{5}$. Ainsi, à première vue, on en perçoit mieux l'utilité que l'agrément. La rhétorique semble prendre le pas sur toute autre visée. Tandis que, dans un roman comme Cleveland, la majeure partie du métadiscours porte sur la meilleure forme à donner à la narration, dans Le Doyen il est moins question de la manière dont on fait un bon récit que de la manière dont on fait un bon discours.

3 Le roman est fondé sur une série de débats autour de dilemmes moraux, et se met souvent en place un véritable tribunal, où le doyen fait d'ordinaire figure de juge. Là, 
les discours rapportés prennent deux formes principales. Les uns appartiennent à la catégorie des discours délibératifs, visant à décider d'une action à venir. Les autres, qui sont des récits d'événements, peuvent être rapprochés des discours judiciaires, portant sur des faits passés, en forme d'apologies ou de réquisitoires et susceptibles eux aussi de conduire à une prise de décision. C'est ainsi, par exemple, que le récit fait par Georges au premier livre pour justifier auprès du doyen sa conduite frivole à Paris avec Rose est qualifié a posteriori d'" apologie ».

Comme dans un manuel de rhétorique à l'usage des collèges jésuites (pensons par exemple au Candidatus rhetoricae de Pomey ${ }^{6}$ ), les discours sont fréquemment suivis de commentaires, qui en soulignent les articulations, et les effets produits chez l'auditeur dans leurs différentes parties. Ainsi, après avoir rapporté un discours de son frère Georges au livre VII, le doyen entreprend de le commenter point par point :

La première partie de son discours m'avait causé de la joie, et je l'aurais interrompu volontiers pour louer l'intérêt qu'il avait pris aux larmes de ma belle-sœur. Surpris ensuite de la naissance de sa passion, j'avais été prêt encore à l'interrompre pour lui faire un reproche de n'avoir pas mieux veillé sur des mouvements de cœur que j'aurais traités de coupables et d'illégitimes; mais en entendant qu'il s'en applaudissait, et qu'au lieu de les combattre, il ne parlait que de les nourrir avec complaisance pour chercher tôt ou tard à les satisfaire. Le ressentiment de me voir si peu ménagé par cette indigne confidence, autant que l'intérêt de la vertu, me fit prendre un ton que j'aurais affecté de rendre encore plus dur, si j'en avais connu de plus propre à lui exprimer mon indignation ${ }^{7}$.

Le commentaire permet d'effectuer rétrospectivement une division dans le discours de Georges, de mettre à nu son architecture. Mais il joue aussi peut-être un rôle d'aidemémoire : le lecteur qui se serait laissé distraire dans la lecture d'un roman aussi long ne risque de manquer aucune information essentielle, puisque le commentaire est en même temps un résumé des éléments principaux du discours. De la même manière, après que le valet de Patrice lui a raconté le mariage de son maître avec mademoiselle de L..., le doyen précise :

J'avais eu le temps, pendant ce récit, de me remettre de toutes les agitations que l'exorde m'avait causées. La retraite modeste de mademoiselle de L... réparait un peu la témérité de son mariage [...]. (VIII, p. 242)

Le substantif exorde pose le récit en discours rhétorique, et, ici comme souvent, les points essentiels sont réduits à des syntagmes nominaux : la retraite modeste, la témérité de son mariage, qui fonctionnent comme des intitulations a posteriori, indiquent les articulations d'un texte qui exhibe sa rhétorique.

5 Qu'en est-il dès lors du sous-titre alléchant du Doyen, qui le décrit comme une « Histoire morale [...] ornée de tout ce qui peut rendre une lecture utile et agréable »? Les discours qui font le corps de ce roman sont austères, le romanesque paraît sacrifié à la rhétorique. L'ouvrage serait-il donc entièrement soumis à l'utilité ? Mais de quel genre d'utilité s'agit-il, précisément, si la rhétorique devient l'objet même du roman?

\section{Le refus du romanesque}

\subsection{Austérité du contenu : le refus de la variété}

Dès les premières pages du roman, le doyen s'emploie à faire des portraits précis de ses frères, de sa sœur et des différents acteurs du drame. Mais s'agit-il de les montrer en 
train de discourir, l'effort de précision semble brusquement achopper, et rien de ce qui fait habituellement le charme du discours rapporté ne transparait.

7 Les discours sont en effet très peu caractérisés : nulle recherche de réalisme dans le discours direct, nul effort de la part du doyen, le narrateur, pour imiter un quelconque idiolecte. Nous sommes bien loin des romans de Marivaux, où l'on entend Mme Dutour jurer, M. de Climal se contorsionner en discours alambiqués et Marianne minauder, bref, où les personnages sont largement définis par leur façon de parler. Dans Le Doyen de Killerine, il importe peu, sur un plan stylistique, qu'un discours soit assumé par un personnage secondaire ou par le doyen lui-même: sans le verbe d'attribution et le contexte, il serait difficile d'attribuer à tel ou tel une réplique ou une autre. Cette proximité entre les différentes voix entraîne une grande fluidité dans le passage d'un type de discours à l'autre : la survenue du discours direct n'introduit pas de rupture stylistique nette, puisque les interlocuteurs du doyen s'expriment comme le doyen luimême.

Du discours indirect au discours indirect libre, il n'y a bien souvent qu'un changement dans le système de la subordination, et du discours indirect libre au discours direct s'effectue presque exclusivement une transposition vers un système déictique. En voici un exemple (il s'agit d'un dialogue où Georges tente de convaincre le doyen de le laisser épouser Sara, la femme de Patrice, dont ce dernier veut divorcer) :

[Georges] parut recevoir ma visite avec quelque embarras. Il me fit des excuses d'avoir passé une semaine entière sans me voir et [...] il passa tout d'un coup à me raconter le succès de ses soins. L'aversion de Patrice, me dit-il, était un caprice qu'il ne pouvait comprendre; et quelques jours de connaissance lui ayant fait découvrir tout le mérite de notre malheureuse belle-sœur, [...] il voulait à toutes sortes de prix lui procurer un sort plus heureux. [...] depuis ce temps-là il l'avait entretenue dans le même calme, en l'assurant qu'il était uniquement occupé de son bonheur [...]. En effet, continua-t-il d'un air encore plus embarrassé, je puis lui en offrir une voie infaillible; et si je tarde à la lui proposer, c'est pour lui laisser le temps de revenir par degrés de ses longues agitations. (VII, 214)

La vitesse du récit ralentit progressivement, jusqu'à rejoindre la temporalité de la scène. À une séquence de discours narrativisé («il me fit des excuses») succèdent en effet une séquence de discours indirect libre et une séquence de discours direct (à partir de : "En effet, continua-t-il d'un air encore plus embarrassé »). La parole au discours direct n'a que très peu de relief et de charme, elle ne se distingue de ce qui précède ni par la syntaxe ni par le vocabulaire. Il est facile de passer la parole aux autres, quand on fait parler les autres comme soi-même. Même quand il feint de laisser parler ses frères, c'est toujours la voix du doyen que nous entendons.

9 La proximité peut être telle qu'elle rend la lecture difficile. C'est par exemple le cas dans cette discussion entre le doyen et Georges, qui refuse d'épouser Mlle d'Anglesey après lui avoir fait un enfant :

Ses raisons furent celles qui m'avaient déjà fait pitié dans la bouche de ses quatre amis. Il ne s'était engagé par nulle promesse; il n'avait pensé qu'à se faire un amusement en Irlande; pouvait-on prétendre que la faiblesse d'une fille lui acquît des droits sur la fortune et sur la liberté d'un homme? Étranges arguments, qui supposent toujours que la faute soit uniquement du côté du sexe le plus faible, et qu'il n'y ait de réparation nécessaire dans ces sortes d'aventures que pour ce qu'il a plu au monde de nommer l'honneur des femmes! (XII, p. 385)

Ici, le lecteur doit redoubler d'attention pour comprendre qui parle. Lorsque nous lisons : «Étranges arguments... », à la suite d'une longue séquence au discours indirect 
libre, nous pouvons en effet avoir un moment de léger flottement : ce propos est-il la suite de ceux de Georges, ou le doyen vient-il de reprendre la parole? En somme, refuser la caractérisation par la syntaxe et le vocabulaire est peut-être un moyen de dire que c'est sur le fond, non sur la forme, que se jouent les oppositions entre les personnages, et de forcer le lecteur à être attentif aux arguments, afin de pouvoir discerner l'origine des paroles. Exigeant avec ses frères et sa sœur, le doyen l'est également avec son lecteur.

Que se passe-t-il alors quand un personnage parle en des termes qui ne sauraient être ceux du doyen? Ce sont des choses qui arrivent, et la solution est aussi simple que radicale : la censure. Les discours censurés sont de trois ordres : les discours insultants, les discours licencieux et les discours descriptifs.

11 Ainsi, au septième livre, le doyen rapporte en ces termes une querelle qu'il a eue avec Patrice :

il me reprocha de l'avoir perdu par mes conseils; et joignant à ce reproche les noms les plus odieux, il jura que ma vie lui répondrait de celle de son amante. À quelques mots que je repris timidement pour ma justification, il continua de répondre par un torrent d'injures; et ses derniers termes furent un adieu terrible par lequel il renonça pour jamais à me voir et à m'entendre. (VII, p. 210)

Les «noms les plus odieux » et le «torrent d'injures » ne sont pas cités. On est loin du plaisir que trouve Marianne, à la même époque, à raconter les échanges passionnés entre la lingère et le cocher.

La censure s'exerce plus souvent encore contre les discours licencieux. Ainsi, il n'y a aucune séquence au discours direct dans la grande scène très théâtrale où Patrice et son amante mademoiselle de L... sont surpris par Sara Fincer au milieu de leurs ébats amoureux. Le doyen se contente de parler, fort vaguement, des «tendres protestations de Patrice» (VII, p. 254), et de dire que Sara entendait Patrice «faire des détails qui ne convenaient qu'à une épouse déclarée " : si ces détails ne conviennent qu'à une épouse, il est compréhensible que le doyen ne veuille pas les rapporter à son lecteur. Certes, un autre argument que celui de la censure vient s'ajouter ici : le doyen n'a pas assisté directement à la scène, et il serait peu probable que Sara ou Patrice lui eussent raconté ensuite le détail des discours tenus à cette occasion. Mais l'impératif premier semble bien être d'éviter les détails trop piquants. De la même manière, l'intransigeant doyen interrompt autoritairement le discours de Patrice au sujet de son amour pour Mlle de L...: :

J'interrompis Patrice au milieu de cette effusion de cœur. Je conçois, lui dis-je, que la connaissance de votre amour peut être nécessaire à l'éclaircissement de vos affaires; mais vous devriez vous épargner ces détails passionnés, qui ne m'apprennent rien que je ne puisse supposer, et que ma profession ne me permet pas d'entendre sans quelque embarras (II, p. 59).

Et c'est également ainsi qu'il interrompt le récit de Rose : «Elle voulait continuer de m'apprendre ce qu'elle avait eu à souffrir de M. le duc de... et de ses autres amants; mais trouvant ce détail inutile à toutes les idées qui m'occupaient, je l'interrompis » (IV, p. 127). Si ce qu'il reproche ouvertement à ce « détail » est d'être « inutile », il laisse entendre que ce qui lui fait peur est d'abord le discours licencieux.

Le dernier type de discours censuré est, justement, le discours perçu comme absolument inutile, car descriptif : le doyen fait profession de refuser absolument la description vaine. C'est pourquoi il transforme ces séquences discursives en discours narrativisés, très faiblement détaillés : 
Le soir, lorsque l'heure du souper nous rassemblait, j'étais obligé d'essuyer les récits éternels de Georges, qui nous racontait tout ce qu'il avait vu; et ceux de Rose, qui n'était pas moins charmée de tous les objets qui l'avaient occupée dans son poste. (I,

p. 26-27)

Bref, un discours qui n'a pas de but est, selon le doyen, non seulement condamnable en soi, mais condamnable dans le système de la narration. En présence du doyen, on n'est pas censé parler pour ne rien dire. Ce faisant, le doyen s'inscrit évidemment contre les conventions du romanesque, et joue à frustrer les attentes du lecteur. Georges et Rose, qui ne connaissent rien à la vie parisienne, se sont peut-être adonnés à des descriptions aussi plaisantes et piquantes qu'Usbek et Rica. Mais le doyen refuse de faire un roman dans le genre des Lettres persanes.

Si, comme l'a montré Jean-Paul Sermain, les héros de Prévost se font fréquemment orateurs et aiment à discourir devant des tribunaux improvisés ${ }^{8}$, la rhétorique du doyen est d'un genre bien particulier : contrairement à un des Grieux, qui cherche à convaincre en suscitant la compassion sur son sort ou en flattant son interlocuteur, le doyen n'essaie guère d'attirer la sympathie, maîtrise mal la captatio benevolentiae, et semble ignorer les pouvoirs de la flatterie, ou en tout cas refuser d'y avoir recours.

\subsection{Austérité de la forme : les tirades conjonctives et les tirades pronominales}

Le caractère fastidieux des discours est souligné par la forme même qui leur est donnée. Dans les deux premiers livres, il y a en effet une profusion de longues tirades au discours indirect, rythmées par la reprise anaphorique de la conjonction de subordination que (" je lui représentai que..., et que..., et que... et enfin que... ») : nous proposons de les appeler tirades conjonctives.

Elles sont très fréquemment employées dans l'argumentation, comme au tout début du roman, où le doyen tente de convaincre son père d'épouser sa propre fiancée :

je lui dis qu'étant encore au-dessous de quarante ans, et jouissant d'une santé parfaite, il était étrange qu'il se fût obstiné à renoncer au mariage ; que c'était un engagement néanmoins qui lui convenait beaucoup plus qu'à moi ; que l'amourpropre ne m'empêchait point d'ouvrir les yeux sur mes imperfections [...]; que la justice que je savais me rendre, et l'estime sincère que j'avais pour elle, me faisaient craindre avec raison qu'elle ne se fît violence pour souffrir ma présence [...]. (I, p. 16)

Le procédé est systématique et mécanique, et se développe au prix d'erreurs. Ainsi, au livre I, quand Georges s'efforce de justifier la conduite que sa sœur et lui ont tenue à Paris, et que le doyen juge frivole :

Georges employait tout son esprit pour donner un tour favorable à ce qu'ils avaient fait ensemble. Il avait cru, me dit-il, que suivant le projet que nous avions formé dès l'Irlande, de marier Rose honorablement ou de la placer auprès de quelque dame de distinction, il était à propos qu'elle se fît voir dans le monde, et qu'elle s'y fît quelques connaissances; qu'il l'avait menée dans cette vue à la promenade et à la comédie ; qu'il n'avait pu empêcher qu'elle n'y fût traitée civilement [...]; qu'au reste il ignorait quel était ce seigneur débauché [...]. (I, p. 30)

Les subordonnées conjonctives dépendent ici en principe du verbe croire, mis en facteur commun, mais le doyen en arrive vite à une incohérence sémantique : * il avait cru qu'il l'avait menée dans cette vue à la promenade et qu'il n'avait pu empêcher qu'elle n'y fût traitée civilement ». La phrase ne fonctionne pas, et il nous faut plutôt supposer qu'un verbe déclaratif a pris le relais. De la même manière, au livre IV, quand le valet 
de des Pesses retrouve le doyen et lui fait un compte rendu de ce qui est advenu à Rose, nous lisons :

il m'apprit que son maître l'avait laissé à Paris, en partant pour l'Allemagne, avec la seule commission de veiller sans cesse à la sûreté de ma sœur ; que ne l'ayant pas perdue de vue, [...] il venait s'informer régulièrement de sa santé, et lui offrir tout ce qu'il était capable d'entreprendre pour son service ; que s'étant retirée [...] dans une chambre fort mal en ordre, où elle s'obstinait à ne voir personne, il avait eu beaucoup de peine à se procurer la permission de la voir, et qu'il me confessait que pour la tromper, après plusieurs tentatives inutiles, il s'était fait annoncer sous le nom d'un de mes domestiques; qu'elle lui avait pardonné ce stratagème [...]. (IV, p. 117)

Ici, c'est la dernière occurrence qui est problématique : «il me confessait que... » ne peut logiquement dépendre de « il m'apprit que », et ne devrait donc pas être précédée de la conjonction que.

Sans aller jusqu'à y voir une stratégie délibérée et retorse de l'auteur implicite pour discréditer le doyen, de telles incohérences prouvent que ces discours sont fort peu travaillés sur un plan stylistique, et pour cause : tout se passe comme si l'impression qu'ils donnent devait être déplaisante. Les tirades conjonctives donnent à l'argumentation une véhémence qui tend à l'agressivité. Et si elles ne peuvent logiquement survenir qu'en discours indirect, elles trouvent une variante très proche dans le discours direct : il s'agit alors de tirades qu'on pourrait appeler pronominales. Le procédé, sous ce double aspect, parvient à saturer le texte.

Vers le début du roman, le doyen adresse à Georges et Rose des reproches en ces termes :

Une fille, dis-je à ma sœur, qui, dans moins de quinze jours, a renoncé à toute bienséance et à toute pudeur; qui va se livrer d'elle-même aux caresses et aux flatteries des hommes, qui se trouve tout d'un coup en liaison avec le seigneur le plus débauché de la cour; un jeune homme [...] qui se rend le ministre des mauvaises inclinations de sa sœur, qui lui ouvre lui-même le chemin de la débauche, qui cherche volontairement à se perdre, et qui entraîne toute sa maison avec lui dans le précipice ; quelle étrange manière de travailler à s'établir en France par les voies de l'honneur et pour la cause de la religion? (I, p. 30)

Immédiatement après, Georges lui répond par une "apologie», et sa défense est rapportée sous la forme d'une très longue tirade conjonctive, dont nous avons déjà cité le début:

Il avait cru, me dit-il, qu[...]'il était à propos qu'elle se fit voir dans le monde [...]; qu'il l'avait menée dans cette vue à la promenade et à la comédie ; qu'il n'avait pu empêcher qu'elle n'y fût traitée civilement [...]. (I, p. 30-31)

À la reprise anaphorique du pronom relatif qui, au sein du discours direct, répond celle de la conjonction que. L'un se situe au niveau de l'histoire et fait partie du discours direct, l'autre au niveau du récit, mais la frontière n'est pas étanche entre les deux, car, encore une fois, le doyen ne fait guère de différence entre les discours rapportés et le reste de son récit.

19 Il est remarquable que ces tirades conjonctives n'interviennent pas seulement dans les discours argumentatifs, mais aussi dans les narrations. Ces dernières gagnent alors une couleur rhétorique, puisque les éléments du récit se transforment en autant d'arguments. C'est ainsi que ce qui pourrait être un simple récit est transformé en une « apologie » en plusieurs points9. 


\section{La rhétorique inutile}

Tout semble donc se passer comme si, au lieu de combiner l'utile et l'agréable, le narrateur, rhétoricien demi-habile, avait à cœur de sacrifier l'agréable à l'utile. Mais, ce faisant, il oublie que le docere ne peut se passer du placere : et en sacrifiant l'agréable à l'utile, il sacrifie, par excès de zèle, l'utile lui-même.

21 La mise en garde contre les dangers d'une certaine rhétorique est parfois tout à fait explicite. Ainsi, au cinquième livre, le discours que fait Georges (alias Tenermill) au doyen pour justifier la conduite de Patrice est nommé « torrent d'éloquence profane » :

[Tenermill] m'interrompit pour me protester avec quelques-unes de ces imprécations galantes qui sont en usage dans le beau monde, que rien n'était si sincère que ses sentiments : que je faisais tort à la religion, en lui attribuant des rigueurs qu'elle n'avait point; que le point d'importance pour de faibles hommes était de rendre à Dieu ce qu'ils lui doivent ; [...] qu'il fallait ou rompre tout à fait avec le monde, ou suivre ses usages. Enfin ce torrent d'éloquence profane ne se serait pas arrêté facilement, si dans le chagrin d'entendre tant de misérables raisonnements, je ne l'eusse interrompu à mon tour pour le prier de changer d'entretien. (V, p. 164-165)

Ici, il ne s'agit pas d'une remise en cause de toute rhétorique, mais de ce que le doyen perçoit comme une insupportable parodie de ce que doit être la rhétorique. L'actio de l'orateur est elle-même dépréciée : Tenermill accompagne son discours « d'un air riant et moqueur », tandis que le doyen a le «ton ferme et sérieux qui convient à la vérité » (p. 164).

Mais la remise en cause de l'utilité des discours en général, y compris de ceux du doyen, peut être sentie dès les premières pages du roman, et comme en dépit de la volonté du narrateur. Il est remarquable que la grande majorité des discours prononcés dans le roman soient des échecs, absolus ou relatifs, et nous sommes loin de la collection des meilleurs endroits de Cicéron. Nous avons déjà cité le premier discours, qui est aussi la première tirade conjonctive. C'est celui que fait le doyen pour convaincre sa fiancée d'épouser son père ( "je lui dis qu'étant encore au-dessous de quarante ans [...], il était étrange qu'il se fût obstiné à renoncer au mariage [...] ») : la débauche d'arguments est inutile, puisque le doyen convainc une convaincue, qui ne fait semblant d'hésiter que par politesse. Le deuxième discours est celui de des Pesses, l'ami de la famille, qui énumère les avantages qu'il y aurait pour les jeunes gens de se rendre à Paris (I, 22). Ce discours prend aussi la forme d'une très longue tirade conjonctive, et là encore, la profusion est peu utile, puisque Georges et Patrice sont déjà convaincus de la supériorité de la France sur la triste Irlande. Parfois, on convainc des réticents, mais alors c'est à tort. C'est le fameux exemple du doyen convainquant son frère Patrice d'épouser Sara, alors que Patrice aime Mlle de L... : cette persuasion mènera in fine à la mort de Sara.

Et nous en arrivons au cas, fréquent, du discours proprement inutile. C'est alors que le comique fait irruption dans le roman, et que la vanité des harangues est mise en évidence le plus clairement. Une scène de ce genre se trouve au livre II, et se joue entre Patrice et M. de L..., qui veut le faire renoncer à sa fille. M. de L... menace Patrice avec une épée, et lui dit qu'il mourra s'il ne la dégage pas sur-le-champ de toutes ses promesses. Patrice répond alors par une longue tirade conjonctive :

je répondis avec une fermeté à laquelle M. de L... ne s'attendait pas, qu'il était le maître de ma vie, puisque je me trouvais sans défense; qu'avec la possession du 
cœur auquel il voulait me faire renoncer, la mort n'avait rien qui me parût terrible [...]; mais que, s'il voulait écouter la raison, il me traiterait peut-être avec plus d'humanité ; que ma naissance, et l'honnêteté de mes vues et de mes sentiments, ne méritaient pas son mépris ni sa haine... Il m'interrompit, en jurant de nouveau qu'il allait m'enfoncer son épée dans le sein [...]. (II, p. 74)

Cette scène n'a aucune fonction dramatique directe : elle ne semble servir qu'à montrer l'échec de la parole rhétorique. D'ailleurs, Patrice finit par jurer qu'il dégage Mlle de L... de toutes ses promesses et qu'il renonce à ses droits sur elle, mais se convainc par la suite que son serment n'a aucune valeur et qu'il n'est donc pas tenu de le respecter. C'est dire le peu d'importance de la parole. Songeons encore à la harangue que tient le doyen à la libertine Mme de S..., et qui ne la convainc absolument pas :

prenant occasion de mes reproches pour y joindre ce que je connaissais de plus puissant dans les principes de la religion et de la morale, je me flattais, après une longue harangue, qui fut écoutée avec la même affectation de douceur et de complaisance, qu'on avait pris du moins quelque goût à m'entendre, et j'eus la crédulité de l'interpréter comme la première marque du succès que j'avais désiré. [...] En m'écoutant, madame de S... m'avait trouvé fort comique d'entreprendre sa conversion [...]. (IX, p. 295)

Le mot «comique » est formulé, et il est probable que le lecteur se sera senti plus proche, en l'occurrence, de madame de S..., qui écoute le discours en spectateur amusé, que du malheureux doyen.

La mise en scène du discours elle-même tend à montrer l'échec de la parole. Les personnages, qui s'installent volontiers dans le cadre d'un procès, se demandent les uns aux autres de s'écouter sans s'interrompre afin de pouvoir se livrer à de longues harangues bien structurées. Mais le contrat n'est jamais respecté bien longtemps. Au livre XII, Georges prie encore son frère « de l'écouter sans l'interrompre » (p. 387). Mais un peu après, le doyen rompt l'engagement qu'il a pris : "malgré la promesse que j'avais faite de l'écouter sans interruption, je me crus obligé de lever un doute », puis : «je me sentis le sang assez ému pour oublier encore la promesse que je lui avais renouvelée de ne pas l'interrompre » (p.388), et enfin : " passez, passez, interrompisje ». De la même manière, quand Georges dit au doyen son envie d'épouser lui-même Sara, la femme de Patrice, son discours est introduit ainsi : " il me conjura de l'écouter sans l'interrompre » (VII, p. 220). Le début du discours est ensuite qualifié par le doyen d'exorde: nous sommes bien dans un cadre rhétorique. Mais le contrat est vite rompu : « je l'arrêtai ici brusquement, malgré la promesse que je lui avais faite de l'écouter sans l'interrompre»(p.221). Aussi Georges se trouve-t-il forcé de supplier son frère pour avoir la parole :

Dans le zèle amer qui m'animait, j'aurais continué de l'accabler de reproches, et je n'aurais pas manqué d'y joindre les plus vives menaces, [si Georges] ne se fût jeté presque à mes pieds, pour renouveler les instances qu'il m'avait faites de l'écouter. Je l'interrompis encore néanmoins: Non, lui dis-je [...]. Cependant comme ses efforts ne diminuaient pas pour obtenir d'être écouté, et que l'embarras où il était, joint à la posture humiliée où je voyais devant moi un caractère si fier, eurent quelque pouvoir pour me fléchir, je consentis à l'entendre. (VII, p. 221)

Un peu plus loin, de nouveau, le doyen n'y tient plus : «ce récit devenant trop long pour mon impatience, je l'interrompis » (p. 223).

En somme, le roman pourrait être vu comme une série d'échecs de la parole rhétorique : on convainc des convaincus, on convainc à tort, on échoue à convaincre, on méprise l'éloquence d'autrui sans s'interroger sur les effets de la sienne propre, on ne respecte pas la parole donnée... Au lieu d'un manuel de rhétorique avec une 
collection d'exemples de discours efficaces, on aurait une collection d'exemples à ne pas suivre, une leçon sur les douteux pouvoirs de la parole.

\section{Le Doyen, vaste discours rhétorique?}

Que penser, dès lors, de la structure d'ensemble du roman? Le Doyen de Killerine peut être considéré comme un long discours contenant des discours: il ressemble à de nombreux égards aux discours des personnages eux-mêmes, comme si ceux-ci étaient une mise en abyme de celui-là.

L'avant-propos est en effet qualifié d'"exorde", et, de même qu'un discours rhétorique, le roman se présente tout entier comme tendu vers un but précis. Ici, contrairement à ce qui se passe dans des œuvres comme Manon Lescaut ou L'Histoire d'une Grecque moderne, l'intention déclarée n'est pas de se justifier, de se défendre contre des accusations, mais de prêcher en vue de bonnes actions futures: c'est "l'envie de se rendre utile", affirme le doyen, qui lui fait prendre la plume, et qui le "fera tourner les événements les plus profanes à l'instruction de la jeunesse, à l'édification de tous les âges et de toutes les conditions, et par conséquent à l'honneur du Ciel et à l'avantage de la société humaine» (p.14). De même que la plupart des discours du doyen sont délibératifs, de même le roman est tourné vers l'avenir.

Le refus obstiné du détail et des scènes licencieuses, qui gouverne la manière dont le doyen rapporte les discours des autres, préside aussi à son propre récit. D’où la multiplicité des formules d'excuse quand il s'agit pour le doyen de passer outre ses principes : « je ne m'arrête à ce détail que pour justifier ma propre conduite » (I, p. 31). À l'instar d'un discours rhétorique, le roman se présente comme construit à rebours, à partir de sa " fin", dans les deux sens du terme : construit à partir de sa conclusion, il est également construit à partir de son objectif. C'est pourquoi le discours d'excuse prend souvent la forme de prolepses, qui montrent que le propos tend à un but bien précis: "Je tirerais le rideau sur cette scène profane, si elle ne se trouvait liée nécessairement à l'histoire de mes frères " (IX, p. 296). C'est bien ici une démarche d'orateur, qui veut signaler à l'auditoire qu'il n'oublie jamais où il veut en venir. Quintilien, dans son Institution oratoire, donne explicitement le conseil d'user de ce genre de formules :

Si c'est un exposé des faits un peu long et plutôt compliqué qui va suivre l'exorde, il sera bon précisément d'y préparer le juge, comme Cicéron l'a fait assez souvent et, particulièrement, dans le passage suivant : "J'irai chercher un peu loin en arrière le début de ma démonstration; je vous demande, juges, de ne pas m'en savoir mauvais gré, car lorsque vous connaîtrez le début, il vous sera plus aisé de comprendre la fin $^{10} »$.

Autrement dit, le récit d'événements «légers» est d'emblée justifié par les conséquences futures de ces événements, et la prolepse est alors une évocation discrète qui permet d'esquisser une téléologie de la narration ${ }^{11}$. Ce motif, qu'on pourrait appeler le "topos de la prolepse légitimante", n'est certes pas propre à Prévost. Ainsi la Marianne de Marivaux y a-t-elle également recours : «Tous ces détails sont ennuyants, déclare-t-elle, mais on ne saurait s'en passer; c'est par eux qu'on va aux faits principaux. » Mais la logique du doyen est inverse. Ce qu'il craint (ou feint de craindre) n'est pas d'être accusé d'ennuyer son lecteur, mais au contraire de trop vouloir le distraire. C'est le détail piquant qui est soupçonné, et qui doit être justifié. Jamais le doyen ne redoute d'ennuyer son lecteur. 
Peut-on, alors, mettre en rapport la thématisation croissante de l'échec de la parole rhétorique au sein du roman avec le destin du roman lui-même? Autrement dit, peuton aller jusqu'à affirmer que, de même que les discours rapportés à l'intérieur du roman sont mis en échec, ridiculisés ou menacés d'interruption, de même le vaste discours que constitue l'ensemble du roman est discrédité, ou menacé d'interruption ?

De fait, d'une part, quand il censure leurs discours injurieux, licencieux ou inutiles, le doyen fait à Rose, à Patrice et à Georges exactement ce que les jésuites ont fait aux auteurs de romans dans les années 1735 : il leur impose le silence, ou du moins une retenue qui met en péril l'univers romanesque. D'autre part, le paradoxe pour ce roman qui s'affiche comme construit en fonction de sa fin (au double sens, donc, de " conclusion » et d'« objectif moral »), c'est qu'il est en même temps largement écrit, pour reprendre une expression de Marc Escola, «dans l'ignorance de sa fin ${ }^{12}$ ». Aurelio Principato a analysé la multiplicité des prolepses déceptives dans Le Doyen de Killerine, qui indiquent, selon lui, des revirements dans la pensée de Prévost entre 1735 et 1740. Un exemple frappant en est l'étrange destin de Patrice. Son portrait fait par le doyen au premier livre laisse entendre qu'il finira par se cloitrer, mais cette anticipation est contredite par la fin du roman : Patrice finit ses jours en homme marié, et non dans un couvent ${ }^{13}$. De même que les personnages doivent composer avec leurs auditeurs quand ils font un discours et infléchir le cours de leur argumentation, de même le romancier doit composer avec son public, et son roman dévie de la trajectoire qu'il semblait s'être fixée. Si la présence de prolepses est certainement liée à une démarche rhétorique, leur mise en échec est peut-être un témoignage de la crise de la démarche rhétorique.

31 Mais jusqu'où faut-il pousser la comparaison? Faut-il vraiment voir dans la mise en scène des malheurs de la rhétorique une métaphore des malheurs du roman lui-même? Nous ne nous aventurerons pas à donner de réponse assurée à la question. Terminons simplement en relisant dans cette perspective la dernière scène du roman. L'ultime discours est celui que le doyen fait à Georges (Tenermill), pour le convaincre de s'éloigner de sa maîtresse doña Figuerrez et d'épouser Mlle Anglesey, dont il vient d'avoir un enfant. Or, ce discours est d'un genre particulier :

J'allais continuer, et je confesse que dans le trouble où j'étais, je parlais presque au hasard, en suivant la seule impression qui me restait de la méthode que nous avions employée avec doña Figuerrez. [...] Mon discours, quoique peu réfléchi, produisit plus d'effet que je n'osais en attendre. (XII, p. 397-398)

Cette parole "au hasard», si elle doit être comprise comme une parole guidée par la Providence, s'oppose en même temps à la parole «méditée à loisir » qui dominait tout le début du roman ${ }^{14}$. Si elle fonctionne, c'est justement parce qu'elle n'est pas guidée par les lois de la rhétorique. Et à cette parole hasardeuse succède le silence. C'est l'ultime paragraphe du roman :

Tenermill allait répondre, et j'ai toujours été persuadé que cédant déjà à la force des circonstances, il ne cherchait plus que des expressions pour nous expliquer le changement de ses idées. Mais la comtesse le prévint, en lui présentant son fils [...]. Tenermill regarda quelques moments cet enfant, qu'on lui faisait reconnaître pour son fils. Il jeta ensuite les yeux sur sa femme. Ses regards s'animaient par degrés. Son visage même s'enflamma. S'il ne lui échappait point un seul mot, il semblait que ce fût la confusion, ou quelque autre sujet de crainte, qui lui liât la langue. Enfin, penchant la tête sur le visage de son fils, il le serra un moment de ses lèvres; et dans le même mouvement, il se leva d'un air passionné pour embrasser mille fois sa femme. Elle ne répondit à des caresses si chères que par des larmes de tendresse, auxquelles nous mêlâmes les nôtres. (XII, p. 399) 
Les « expressions » rationnelles traduisant des «idées » se dérobent aux personnages, et leur langue est désormais «liée ». Les larmes et les caresses prennent la place du discours : le silence du romancier coïncide avec celui des personnages.

En somme, s'il est difficile de donner tout à fait tort à Desfontaines lorsqu'il écrit au sujet du Doyen : « si vous exceptez les agrémens du stile, \& la vivacité des peintures, [...] tout l'Ouvrage n'est à mon gré qu'une masse informe, ou l'on ne trouve pas assurement, comme l'Auteur le promet sans façon dans son titre, tout ce qui peut rendre une lecture utile \& agréable ${ }^{15}$ », il semble qu'on puisse voir dans le sous-titre du roman, «Histoire morale [...] ornée de tout ce qui peut rendre une lecture utile et agréable », une certaine ironie, le clin d'œil d'un auteur qui sait pertinemment que les prétendus " ornements » de son roman sont d'une austérité remarquable, et que l'utilité morale de l'ouvrage est loin de s'imposer clairement au lecteur. Plutôt que de voir dans Le Doyen de Killerine un roman rhétorique, on peut $\mathrm{y}$ voir un roman où le questionnement sur la rhétorique prend progressivement de l'ampleur, à mesure que l'œuvre elle-même dévie de la trajectoire qu'elle semblait s'être fixée.

\section{NOTES}

1. Voir J. Sgard, Vie de Prévost (1697-1763), Laval, Presses Universitaires de Laval, 2006, p. 142.

2. Voir P. Stewart, "Prévost et son Cleveland: essai de mise au point historique ", Dix-huitième siècle, 1975, p. 181-208.

3. L'expression est également de J. Sgard, op. cit., p. 143 et suivantes.

4. Ibid.

5. Sur les rapports de Prévost avec la culture rhétorique, voir J.-P. Sermain, Rhétorique et roman au XVIII' siècle : l'exemple de Prévost et de Marivaux (1728-1742), Oxford, The Voltaire Foundation, 1985.

6. François Pomey, Candidatus rhetoricae, seu Aphtonii progymnasmata, Lyon, Antoine Molin, 1661. Le texte a ensuite été remanié par Jouvancy en 1710, et traduit partiellement par Henri Ferté, L'Élève de rhétorique [1834], Paris, Hachette, 1892.

7. Prévost, Le Doyen de Killerine [1735-1740], éd. A. Principato, dans Euvres, dir. Jean Sgard, Grenoble, Presses universitaires de Grenoble, 1978, t. 3, livre VII, p. 221. Toutes nos références sont à cette édition, en attendant l'édition à paraître dirigée par Colas Duflo, Audrey Faulot et Érik Leborgne.

8. Voir J.-P. Sermain, op. cit., notamment p. 44-49.

9. Notons que les tirades conjonctives se font plus rares dès le troisième livre. Nous comptons onze tirades fondées sur une énumération de plus de quatre que dans le premier livre, neuf dans le deuxième, et seulement une dans le troisième et deux dans le quatrième. Comment expliquer cette soudaine raréfaction? On pourrait penser que Prévost a suffisamment assis son personnage dans son rôle moralisateur et qu'il n'a plus besoin de recourir à une forme aussi pénible pour le lecteur. Mais les tirades conjonctives ne sont pas le propre du doyen, elles se trouvent également, par exemple, dans le récit de Patrice. Il nous semble difficile de comprendre cette raréfaction autrement que par un changement stylistique de Prévost lui-même. Ce changement brusque pourrait éventuellement nous conduire à remettre en question l'hypothèse communément admise sur la date de rédaction, selon laquelle Prévost aurait eu déjà en réserve la deuxième 
partie en 1735, au moment où il publiait la première. Ce changement stylistique pourrait au contraire laisser penser que Prévost a rédigé la deuxième partie plus tardivement.

10. Quintilien, Institution oratoire (IV, 1, 79), trad. J. Cousin, Paris, Les Belles Lettres, 1976, t. 3, p. 38.

11. C'est plus généralement un trait des narrateurs prévostiens. Cleveland le répète ainsi à l'envi (Prévost, Le Philosophe anglais, ou Histoire de M.Cleveland, éd. J.Sgard et P.Stewart, Paris, Desjonquères, 2003, p. 106, 128, 374) : «C'est une circonstance de ma vie que je veux expliquer avec soin, parce que, quelque légère qu'elle ait été dans son origine, elle a donné depuis naissance à des événements si considérables qu'ils composent la partie la plus intéressante de mon histoire »; «Ce n'est pas sans raison que je fais ici cette remarque, et que j'ai rapporté quelques légères circonstances de mon histoire qui y ont donné occasion »; "Je ne me serais pas tant étendu sur un événement si léger, s'il n'eût produit peu de temps après des effets si terribles que mon sang se glace encore de l'obligation où je me suis mis de les raconter "; "Cet événement, dont j'aurais pu me dispenser d'interrompre ma narration s'il ne s'y rapportait par ses suites $[\ldots] »$.

12. Voir notamment M. Escola, "Longueur de Cleveland», dans J.-P. Sermain dir., Cleveland de Prévost. L'Épopée du XVIII siècle, Paris, Desjonquères, 2006, p. 181-203; "Le clou de Tchekhov, retours sur le principe de causalité régressive", dans M.Escola, J. Herman, L. Omacini, P. Pelckmans et J.-P. Sermain dir., La Partie et le tout : la composition du roman, de l'âge baroque au tournant des Lumières, Louvain/Paris/Walpole, Peeters, 2011, p. 107-117.

13. Dans son article « De l'effet appétissant des prolepses narratives " (La Partie et le tout, op. cit., p. 567-580, ici p. 576-577), Aurelio Principato explique ce changement en disant qu'en 1735, «Prévost devait réparer les suites provoquées par les premiers tomes du Cleveland. Lorsqu'il écrit la fin du Doyen de Killerine, quatre ou cinq ans plus tard, beaucoup d'eau a coulé sous les ponts de la Seine et, en tout état de cause, la proscription des romans rend très relatif ce genre de préoccupations ».

14. Par exemple : « Ce discours, que Georges avait sans doute médité à loisir... » (I, 33).

15. Desfontaines (Guillaume François Fouques Deshayes), Observations sur les écrits modernes, t. 2, Paris, Chaubert, 1736, lettre du 22 juillet, p. 96.

\section{AUTHOR}

\section{LISE CHARLES}

Université de Nantes 